\title{
AVALIAÇÃO DA ACESSIBILIDADE DO PORTAL IFRN À LUZ DO E-MAG E DO WCAG SAMURAI
}

\author{
G. M. M. Gurgel'1, L. O. Medeiros' e L. S. N. T. Moura'
}

${ }^{1}$ Instituto Federal do Rio Grande do Norte - Campus Mossoró

giovane.gurgel@ifrn.edu.br

Artigo submetido em outubro/2011 e aceito em março/2012

\section{RESUMO}

Cada vez mais, a internet é utilizada como meio de comunicação universal, provendo acesso a serviços essenciais a qualquer cidadão que utilize um computador conectado à rede. Assim como os prédios que oferecem atendimento à população precisam estar de acordo com as normas de acessibilidade, o mesmo deve acontecer com os sites de instituições públicas, ou seja, o conteúdo deve estar acessível para os mais diferentes perfis de usuários. Este trabalho tem como objetivo apresentar a avaliação de acessibilidade do portal IFRN observando dois modelos de acessibilidade web: e-MAG 2.0 (iniciativa do Departamento de Governo Eletrônico que adapta o WCAG 1.0 para os sites institucionais) e o WCAG Samurai (proposta independente, alternativa ao WCAG 2.0). Os resultados apontam que o portal IFRN apresenta conformidade a algumas diretrizes de acessibilidade web, mas que ainda há ocorrências que precisam ser corrigidas para o pleno atendimento do nível de acessibilidade de prioridade 1 do e-MAG 2.0. Além de ser mais prático, o WCAG Samurai também amplia a discussão ao explorar novas questões que não estão presentes nas versões do WCAG. Por fim, novas pesquisas são propostas, e seguindo os princípios do e-MAG e WCAG Samurai, são indicadas algumas sugestões para melhorar a acessibilidade do portal IFRN.

PALAVRAS-CHAVE: acessibilidade web, e-MAG, WCAG Samurai.

\section{ACCESSIBILITY ASSESSMENT OF IFRN PORTAL ACCORDING TO E-MAG AND THE WCAG SAMURAI}

\section{ABSTRACT}

Increasingly, the Internet is used as a universal medium, providing essential services to any citizen who uses a computer connected to the network. Like the buildings that provide care to the population must comply with the accessibility standards, the same should happen with the sites of public institutions, i.e. the content should be accessible to many different user profiles. This paper aims to present the assessment of accessibility of the IFRN portal according to two models of web accessibility: e-MAG 2.0 (initiative of the Department of Electronic Government to adapt the WCAG 1.0 to the institutional sites) and the WCAG
Samurai (independent proposal, alternative to the WCAG 2.0). The results indicate that the IFRN portal is according to some web accessibility guidelines, but there are still occurrences that need to be corrected to fully meet the accessibility priority 1 of e-MAG 2.0. Besides being more practical, the WCAG Samurai also broadens the discussion to explore new issues that are not present in versions of WCAG. Finally, further research is proposed, and following the principles of eMAG and WCAG Samurai are given some suggestions to improve the accessibility of the IFRN portal.

KEY-WORDS: web accessibility, e-MAG, WCAG Samurai. 


\section{AVALIAÇÃO DA ACESSIBILIDADE DO PORTAL IFRN À LUZ DO E-MAG E DO WCAG SAMURAI}

\section{INTRODUÇÃO}

Com o uso da Internet, o acesso a serviços oferecidos pelos governos vai além dos prédios das repartições públicas. O compartilhamento de informações promovido por inúmeras aplicações na World Wide Web (WWW) permite que praticamente todo cidadão com acesso à rede seja capaz de ser atendido pelas políticas governamentais. O resultado é uma efetiva participação popular, promovendo democracia e maior inclusão social.

Assim como os prédios que oferecem atendimento à população precisam estar de acordo com as normas de acessibilidade, o mesmo deve acontecer com os sites de instituições públicas, ou seja, o conteúdo deve estar acessível para os mais diferentes perfis de usuários. Um site pode ser definido como acessível quando não apresenta nenhum tipo de barreira que impeça pessoas com qualquer deficiência, seja ela física ou mental, de desfrutar, interagir e entender de forma completa todo o conteúdo oferecido pela página. Acessibilidade agrega novas camadas de significados ao site (CLARK, 2002).

De acordo com dados do censo da Web.br realizado pelo Comitê gestor da Internet no Brasil, 98\% das aproximadamente 6,3 milhões de páginas do governo eletrônico brasileiro não apresentam nenhum tipo de conformidade com os padrões de acessibilidade, enquanto apenas $2 \%$ apresentam algum tipo de conformidade. Esses padrões são elaborados pelo World Wide Web Consortium (W3C) - Web Content Accessibility Guidelines (WCAG) 1.0 e 2.0 - e oferecem diretrizes que os desenvolvedores devem observar ao construir sites mais acessíveis. A análise feita por esse trabalho apresentará uma avaliação da acessibilidade do portal do Instituto Federal do Rio Grande do Norte (IFRN) com base no e-MAG 2.0 (iniciativa do Departamento de Governo Eletrônico que adapta o WCAG 1.0 para os sites institucionais) e no WCAG Samurai (proposta independente, alternativa ao WCAG 2.0).

Dessa forma, a próxima seção descreve a iniciativa de acessibilidade web idealizada pelo W3C. Em seguida são apresentados o modelo de acessibilidade web do governo brasileiro - e-MAG - e o modelo alternativo ao WCAG 2.0 - WCAG Samurai. Em seguida, a metodologia utilizada é apresentada. A seção seguinte apresenta as avaliações com base nos modelos citados. Por fim, a conclusão descreve as considerações finais e propõe novos trabalhos.

\section{WEB ACCESSIBILITY INITIATIVE (WAI)}

De acordo com Tim Berners-Lee (diretor do W3C e inventor da WWW), o poder da Web está na sua universalidade, ou seja, todos podem acessar independente do hardware, software, linguagem, cultura, localização ou de alguma deficiência ${ }^{1}$. Apesar disso, a tecnologia também pode impor barreiras caso não tenha sido bem projetada. Diante disso, as recomendações do $\mathrm{WAI}^{2}$ servem como guia para todos que produzem e acessam conteúdos na Internet.

\footnotetext{
${ }^{1}$ http://www.w3.org/standards/webdesign/accessibility

2 http://www.w3.org/WAl/

HOLOS, Ano 28, Vol 1
} 
Criado desde 1997, o WAl vem desenvolvendo muitos trabalhos que conscientizam sobre a importância da acessibilidade web assim como na elaboração de modelos que auxiliam organizações a desenvolverem conteúdos acessíveis na Web (SLOAN et al., 2006).

Antes do WAI, o foco da acessibilidade era na responsabilidade de quem produzia conteúdo para a Web. Deixava de lado a dependência existente entre vários componentes que estão envolvidos (CHISHOLM; HENRY, 2005):

- Componentes técnicos: especificações técnicas, conteúdo e ferramentas;

- Componentes humanos: fornecedores de conteúdo, usuários finais e ferramentas utilizadas pelos desenvolvedores.

Nesse sentido, o WAl elaborou e mantém um conjunto de recomendações que abordam o conteúdo na Web, ferramentas de navegação (browsers) e ferramentas de autoria. Fazem parte da elaboração dessas recomendações também vários representantes de empresas de tecnologia de vários países.

O WCAG descreve como tornar sites acessíveis a pessoas com algum tipo de deficiência. A versão $1.0^{3}$ foi lançada em 1999 e vem sendo utilizada desde então por diversos governos e organizações que desejam tornar acessíveis seus conteúdos na Web. Segundo Sloan et al. (2006), há algumas falhas no modelo do WCAG que impedem de seu uso efetivo. Entre elas, principalmente a ambigüidade e a complexidade das diretrizes. Isso permite uma interpretação muito ampla e distante da realidade. A versão $2.0^{4}$ (2008) avança no sentido de corrigir os problemas da versão 1.0, mas o que ocorre também é que a Web se torna mais complexa uma vez que surgem novas tecnologias que agregam novas funcionalidades e ao mesmo tempo se tornam novos desafios na garantia da acessibilidade (JESCHKE; PFEIFFER; VIERITZ, 2009).

Complementando o WCAG, lançada em 2002, a recomendação para browsers - User Agent Accessibility Guidelines 1.0 (UAAG) ${ }^{5}$ - e outras ferramentas que exibem conteúdo da Web. A partir dessa recomendação, os browsers deveriam oferecer diferentes formas de navegação, bem como interfaces com outros softwares como plugins (BREWER, 2004).

A recomendação para ferramentas de autoria foi lançada em 2000 - Authoring Tool Accessibility Guidelines (ATAG) ${ }^{6}$. Com essa recomendação, as ferramentas podem ser desenvolvidas tendo em vista a geração de sites acessíveis. Por exemplo, as ferramentas de autoria devem gerar um código válido e orientar o desenvolvedor no atendimento das diretrizes de validação de acessibilidade (BREWER, 2004).

Dessa forma, é responsabilidade de vários agentes para que a acessibilidade possa ser atingida. Por isso demanda a participação de colaboradores não apenas do W3C, mas da comunidade em geral - desenvolvedores e usuários. A seguir, será apresentado o modelo criado pelo governo brasileiro que segue as diretrizes do WCAG.

\footnotetext{
${ }^{3}$ http://www.w3.org/TR/WCAG10/

4 http://www.w3.org/TR/WCAG/

5 http://www.w3.org/TR/WAI-USERAGENT/

${ }^{6}$ http://www.w3.org/TR/ATAG20/
} 


\section{E-MAG - ACESSIBILIDADE NO GOVERNO ELETRÔNICO}

O Modelo de Acessibilidade de Governo Eletrônico (e-MAG) é um projeto do Governo Federal que faz parte da política de Governo Eletrônico e consiste em recomendações para a construção ou adaptação dos sites do governo brasileiro (LARA et al., 2010) no que diz respeito à acessibilidade. Outros países também desenvolveram suas próprias diretrizes de acessibilidade para sites institucionais, por exemplo, em 2007, o Thailand Web Content Accessibility Guideline (Th-WCAG) que adaptou a versão 2.0 do WCAG às necessidades do governo da Tailândia (MITSAMARN; GESTUBTIM; JUNNATAS, 2007).

O e-MAG segue o padrão internacional de acessibilidade web WCAG 1.0. Oferece ao desenvolvedor um guia completo de metodologias a serem aplicadas para criar ou reconstruir um site acessível. $O$ atendimento às diretrizes de acessibilidade está associado a níveis que identificam se o site é menos acessível - A - até mais acessível - AAA. Lançado em 2005 na sua versão 2.0, o e-MAG abrange duas esferas diferentes do conceito de acessibilidade: a cartilha técnica e a visão do cidadão (BRASIL, 2005a, 2005b):

- Cartilha Técnica: Especifica as normas que devem ser seguidas por aqueles que desenvolvem os sites da web. Contém informações técnicas, que permitem a implementação do modelo e-MAG. Dessa forma, bem como o WCAG 1.0, se divide em prioridades que vão de um a três. Sendo a primeira as exigências básicas e obrigatórias da acessibilidade, a segunda são normas que se implementadas vêm a garantir o acesso às informações do documento e já as de terceira prioridade, são aquelas que se implementadas facilitarão o acesso aos documentos da Web. Suas diretrizes expõem de forma geral os resultados obtidos a partir da implementação correta das normas, desta forma buscam facilitar àquele que está desenvolvendo a compreensão e fixação das recomendações feitas;

- Visão do Cidadão: Compreende um público mais abrangente, incluindo pessoas não técnicas no processo de acessibilidade, permitindo que aja um entendimento intuitivo quando ao que está sendo aplicado. Assim, a visão do cidadão auxilia na compreensão de onde e como cada regra descrita irá funcionar, é dividida em quatro áreas:

o Percepção: Diz respeito à parte visual, a apresentação do conteúdo e da parte gráfica do site, considerando as imagens, sons e outras opções de multimídia;

o Operação: Visa à manipulação do conteúdo, garantindo ao usuário o controle sobre a navegação no site. Além de assegurar formas alternativas de acesso às informações utilizando técnicas e metodologias diferenciadas para navegar;

- Entendimento: É a área responsável pela compreensão de todo o conteúdo disponibilizado no site, de forma que todo o público que acessa o site possa entender com facilidade as informações apresentadas;

o Compatibilidade: Compreende a necessidade da utilização de tecnologias acessíveis e compatíveis conforme a cartilha técnica demonstra.

As duas visões se tornam um elo entre quem constrói e quem acessa. Através de suas diretrizes, o e-MAG se torna uma versão adaptada do WCAG à realidade brasileira (Quadro 1). Dessa forma, o e-MAG possibilita uma padronização dos sites da internet pertencentes ao governo, promovendo a inclusão social, gerando igualdade de oportunidades na sociedade da 
informação.

Quadro 1: Diretrizes de Acessibilidade no e-MAG e WCAG 1.0.

\begin{tabular}{|c|c|c|}
\hline N. & e-MAG & WCAG 1.0 \\
\hline 1 & $\begin{array}{l}\text { Fornecer alternativas equivalentes para } \\
\text { conteúdo gráfico e sonoro. }\end{array}$ & $\begin{array}{l}\text { Fornecer alternativas equivalentes ao } \\
\text { conteúdo sonoro e visual. }\end{array}$ \\
\hline 2 & $\begin{array}{c}\text { Assegurar que um site seja legível e } \\
\text { compreensível mesmo sem o uso de } \\
\text { formatações. }\end{array}$ & Não recorrer apenas à cor. \\
\hline 3 & $\begin{array}{l}\text { Dar preferência às tecnologias de marcação e } \\
\text { formatação. }\end{array}$ & $\begin{array}{l}\text { Utilizar corretamente marcações e } \\
\text { folhas de estilo. }\end{array}$ \\
\hline 4 & $\begin{array}{c}\text { Assegurar que toda informação seja } \\
\text { interpretada corretamente, com clareza e } \\
\text { simplicidade. }\end{array}$ & $\begin{array}{c}\text { Indicar claramente qual o idioma } \\
\text { utilizado. }\end{array}$ \\
\hline 5 & $\begin{array}{l}\text { Assegurar que todas as tecnologias utilizadas } \\
\text { funcionem, de maneira acessível, } \\
\text { independente de programas, versões e futuras } \\
\text { mudanças. }\end{array}$ & $\begin{array}{l}\text { Criar tabelas passíveis de } \\
\text { transformação harmoniosa. }\end{array}$ \\
\hline 6 & $\begin{array}{c}\text { Assegurar sempre o controle do usuário sobre } \\
\text { a navegação no site. }\end{array}$ & $\begin{array}{c}\text { Assegurar que as páginas dotadas de } \\
\text { novas tecnologias sejam } \\
\text { transformadas harmoniosamente. }\end{array}$ \\
\hline 7 & $\begin{array}{l}\text { Identificar claramente quais são os } \\
\text { mecanismos de navegação. }\end{array}$ & $\begin{array}{l}\text { Assegurar o controle do usuário } \\
\text { sobre as alterações temporais do } \\
\text { conteúdo. }\end{array}$ \\
\hline 8 & $\begin{array}{l}\text { Em casos não contemplados pelas diretrizes } \\
\text { anteriores, utilizar sempre recursos } \\
\text { reconhecidos por instituições com propriedade } \\
\text { no assunto, como tecnologias acessíveis. }\end{array}$ & $\begin{array}{l}\text { Assegurar acessibilidade às interfaces } \\
\text { de usuário incorporadas à página. }\end{array}$ \\
\hline 9 & & $\begin{array}{l}\text { Projetar páginas considerando a } \\
\text { independência de dispositivos. }\end{array}$ \\
\hline 10 & & Utilizar soluções de transição. \\
\hline 11 & & $\begin{array}{c}\text { Utilizar tecnologias e diretrizes do } \\
\text { W3C. }\end{array}$ \\
\hline 12 & & $\begin{array}{l}\text { Fornecer contexto e orientações para } \\
\text { ajudar os usuários a compreenderem } \\
\text { páginas ou elementos complexos. }\end{array}$ \\
\hline 13 & & $\begin{array}{l}\text { Fornecer mecanismos claros de } \\
\text { navegação. }\end{array}$ \\
\hline 14 & & $\begin{array}{c}\text { Assegurar a clareza e a simplicidade } \\
\text { dos documentos. }\end{array}$ \\
\hline
\end{tabular}

O e-MAG possui oito diretrizes, enquanto o WCAG 1.0 possui 14 diretrizes. Algumas diretrizes do WCAG 1.0 estão agrupadas em algumas diretrizes do e-MAG. Mesmo assim, percebe-se que há uma relação quase direta de equivalência entre o padrão adotado no Brasil e o criado pelo W3C. Em 2010 foi realizada uma consulta pública sobre a nova versão do eMAG. Essa nova versão é baseada nas diretrizes do WCAG 2.0 e ainda não foi publicada. A 
seção a seguir apresenta o WCAG Samurai, elaborado pela comunidade de usuários e que seria uma alternativa ao WCAG 2.0.

\section{WCAG SAMURAI}

O WCAG Samurai ${ }^{7}$ foi criado por um grupo de desenvolvedores independentes (CLARK, 2006), buscando aperfeiçoar as regras descritas no WCAG 1.0 ao invés de adotar a versão 2.0 do WCAG. De acordo com a errata, ou se usa o WCAG Samurai ou se usa o WCAG 2.0. Foi uma reação de desenvolvedores que consideraram a nova versão do WCAG incompatível com o desenvolvimento baseado em padrões já estabelecidos pelo próprio W3C. A proposta da errata é adaptar para a realidade prática dos que constroem páginas as diretrizes de acessibilidade.

Ao fazer correções e destacar os pontos importantes do WCAG 1.0, o WCAG Samurai baseia-se em diretrizes práticas e objetivas, que proíbem ou apontam para a necessidade do uso de alguma regra. Constitui-se numa alternativa enxuta das regras básicas de acessibilidade, que tem por objetivo principal a eficiência dos métodos aplicados, bem como a viabilidade da aplicação de tal método.

O WCAG Samurai estrutura as aplicações práticas para suas diretrizes de modo similar ao WCAG com suas prioridades que vão de um a três. No entanto, considerando a finalidade da errata, as diretrizes de prioridade três não são classificadas como práticas, dessa forma não são recomendadas. O WCAG Samurai possui as mesmas diretrizes do WCAG 1.0, porém, para cada uma dessas diretrizes, o modelo disponibiliza erratas (Quadro 2), que apresentam melhorias ou aperfeiçoam o padrão descrito.

A iniciativa do WCAG Samurai parte da comunidade de usuários e isso também deve ser levado em consideração pelo WAl. Outras pesquisas (SLOAN et al., 2006; JESCHKE; PFEIFFER; VIERITZ, 2009; KELLY et al., 2007) relatam a necessidade de realizar a avaliação de acessibilidade não apenas observando as diretrizes do WCAG, pois podem não ser práticas, permitir ambigüidades e a realidade das aplicações web é mais complexo do que as diretrizes são capazes de tratar.

Quadro 2: Resumo da errata do WCAG Samurai para cada diretriz do WCAG 1.0.

\begin{tabular}{|c|c|c|}
\hline $\mathrm{N}$. & WCAG 1.0 & WCAG Samurai \\
\hline 1 & $\begin{array}{l}\text { Fornecer alternativas equivalentes } \\
\text { ao conteúdo sonoro e visual. }\end{array}$ & $\begin{array}{l}\text { - Deixar o equivalente textual em branco } \\
\text { se o texto imediatamente anterior ou } \\
\text { posterior à imagem cumprir a função de } \\
\text { texto equivalente; } \\
\text { - Não utilizar GIFs animados, arte ASCII, } \\
\text { imagens espaçadoras, ou mapa de } \\
\text { imagens no lado do servidor; } \\
\text { - Sons e vídeos não devem ser ativados } \\
\text { sem que haja intervenção do usuário; }\end{array}$ \\
\hline
\end{tabular}

\footnotetext{
${ }^{7}$ http://wcagsamurai.org
} 


\begin{tabular}{|c|c|c|}
\hline & & $\begin{array}{l}\text { - Vídeos com narração sonora devem ser } \\
\text { legendados. }\end{array}$ \\
\hline 2 & Não recorrer apenas à cor. & $\begin{array}{l}\text { Para conteúdos não defina cores } \\
\text { confusas uma sobre a outra; } \\
\text { - Não usar a cor isoladamente para } \\
\text { transmitir uma informação no } \\
\text { documento. }\end{array}$ \\
\hline 3 & $\begin{array}{l}\text { Utilizar corretamente marcações e } \\
\text { folhas de estilo. }\end{array}$ & $\begin{array}{l}\text { - Qualquer código HTML ou CSS inserido } \\
\text { em uma página por script ou outro } \\
\text { método similar deve produzir uma } \\
\text { página válida quando renderizada; } \\
\text { - Não só o código HTML deve ser válido } \\
\text { na sua página como também seu HTML } \\
\text { deve ser semântico. }\end{array}$ \\
\hline 4 & $\begin{array}{c}\text { Indicar claramente qual o idioma } \\
\text { utilizado. }\end{array}$ & $\begin{array}{l}\text { - Identifique o idioma principal do } \\
\text { documento - de preferência na tag } \\
\text { html que deve estar presente em } \\
\text { documentos XHTML; } \\
\text { - Se uma página é bi ou multilíngue não } \\
\text { especifique um idioma principal já que } \\
\text { nestes casos não existe o idioma } \\
\text { "principal". Especifique o idioma usado } \\
\text { em diferentes partes da página. }\end{array}$ \\
\hline 5 & $\begin{array}{l}\text { Criar tabelas passíveis de } \\
\text { transformação harmoniosa. }\end{array}$ & - Não use tabelas para layout. \\
\hline 6 & $\begin{array}{l}\text { Assegurar que as páginas dotadas } \\
\text { de novas tecnologias sejam } \\
\text { transformadas harmoniosamente. }\end{array}$ & $\begin{array}{l}\text { - Não forneça apresentações ou páginas } \\
\text { alternativas. Construa a página principal } \\
\text { acessível. }\end{array}$ \\
\hline 7 & $\begin{array}{l}\text { Assegurar o controle do usuário } \\
\text { sobre as alterações temporais do } \\
\text { conteúdo. }\end{array}$ & $\begin{array}{l}\text { - "Conteúdos que se movem", rolagens, } \\
\text { movimentações em geral ou animações } \\
\text { não devem ser disparadas } \\
\text { automaticamente sem o controle do } \\
\text { usuário. }\end{array}$ \\
\hline 8 & $\begin{array}{c}\text { Assegurar acessibilidade às } \\
\text { interfaces de usuário incorporadas à } \\
\text { página. }\end{array}$ & - Não há errata para esta diretriz. \\
\hline 9 & $\begin{array}{l}\text { Projetar páginas considerando a } \\
\text { independência de dispositivos. }\end{array}$ & $\begin{array}{l}\text { - Não crie atalhos de teclado } \\
\text { personalizados usando accesskey. Não } \\
\text { use accesskey. }\end{array}$ \\
\hline 10 & Utilizar soluções de transição. & $\begin{array}{l}\text { - Não abra pop-ups ou janelas adicionais e } \\
\text { nem altere a janela corrente sem antes } \\
\text { informar ao usuário; } \\
\text { - Não coloque textos pré-definidos em } \\
\text { caixas e áreas de entrada de textos, } \\
\text { mesmo que sejam removidos por script } \\
\text { quando receberem o foco. }\end{array}$ \\
\hline 11 & Utilizar tecnologias e diretrizes do & - Use XHTML ou HTML para documentos \\
\hline
\end{tabular}




\begin{tabular}{|c|c|c|c|}
\hline & W3C. & $\begin{array}{l}\text { contendo basicamente textos e/ou } \\
\text { gráficos. Variações do HTML } \\
\text { desenvolvidas fora do W3C são } \\
\text { permitidas desde que adequadamente } \\
\text { definidas. }\end{array}$ \\
\hline 12 & $\begin{array}{c}\text { Fornecer contexto e orientações } \\
\text { para ajudar os usuários a } \\
\text { compreenderem páginas ou } \\
\text { elementos complexos. }\end{array}$ & $\begin{array}{l}\text { Não usar frames. Sendo permitido } \\
\text { utilizar iframes; } \\
\text { Não é obrigatório fornecer um mapa do } \\
\text { site ou tabela de conteúdos a menos } \\
\text { que o site não possa ser entendido ou } \\
\text { navegado sem elas. }\end{array}$ \\
\hline 13 & $\begin{array}{c}\text { Fornecer mecanismos claros de } \\
\text { navegação. }\end{array}$ & - Não há errata para esta diretriz. \\
\hline 14 & $\begin{array}{c}\text { Assegurar a clareza e a simplicidade } \\
\text { dos documentos. }\end{array}$ & Não há errata para esta diretriz. \\
\hline
\end{tabular}

\section{METODOLOGIA}

Trata-se de um estudo qualitativo (CRESWELL, 2007) e descritivo (MARCONI; LAKATOS, 2010). A avaliação do portal IFRN ${ }^{8}$ visa descrever o nível de acessibilidade obtido de acordo com as diretrizes dos modelos escolhidos (e-MAG 2.0 e WCAG Samurai). O portal IFRN utiliza o Open Source Content Management System Plone ${ }^{9}$ que segue as diretrizes do WCAG 1.0.

Ao analisar a partir do e-MAG 2.0, será possível verificar o grau de atendimento do portal ao modelo do Governo Eletrônico Brasileiro. O processo consta de três fases (BRASIL, 2005b):

1. Utilizar programas validadores automáticos de acessibilidade;

2. Realizar validação humana, através da navegação do site com programas leitores de tela;

3. Realizar outra validação humana, também através da navegação do site com programas leitores de tela; contudo, desta vez, feita por usuários portadores de deficiência.

O primeiro passo foi realizado através do Avaliador e Simulador de Acessibilidade de Sítios $^{10}$ (ASES) desenvolvido pelo Governo Federal e segue as diretrizes do e-MAG 2.0. 0 segundo passo foi alcançado por meio do software de apoio a deficientes visuais DOSVOX ${ }^{11}$ desenvolvido pelo Núcleo de Computação Eletrônica da Universidade Federal do Rio de Janeiro (UFRJ). Há outros leitores de tela, mas o DOSVOX foi o único compatível com o sistema operacional utilizado na máquina onde foi realizada a análise. O terceiro passo não pode ser realizado; não foi possível entrar em contato com algum portador de deficiência para realizar a validação.

\footnotetext{
${ }^{8}$ http://portal.ifrn.edu.br

9 http://plone.org/

${ }^{10} \mathrm{https}$ ://www.governoeletronico.gov.br/acoes-e-projetos/e-MAG/ases-avaliador-e-simulador-deacessibilidade-sitios

${ }^{11}$ http://intervox.nce.ufri.br/dosvox/
} 
Como o WCAG Samurai é uma errata ao WCAG 1.0 e o e-MAG 2.0 é baseado no WCAG 1.0, será possível obter uma avaliação alternativa e comparar os resultados, verificando se o modelo brasileiro obtém melhores resultados do que o WCAG Samurai ou vice-versa.

Vale salientar também que o e-MAG 3.0 ainda não foi lançado (mas já esteve em consulta pública ${ }^{12}$ ) e é baseado no WCAG 2.0 que, apesar do avanço em comparação ao WCAG 1.0, possui críticas sob o aspecto de sua eficácia e eficiência na garantia de acessibilidade web. Logo, a avaliação com o WCAG Samurai também serve como parâmetro para, futuramente, comparar as vantagens ou desvantagens do e-MAG 3.0.

A avaliação com base no WCAG Samurai foi realizada sem o auxílio de softwares, ou seja, ocorreu de forma manual, analisando todo o código-fonte (1038 linhas) da página inicial do portal IFRN observando o atendimento às suas diretrizes. O pesquisador analisou todos os elementos da página e anotava a ocorrência de cada falha identificada no código-fonte.

\section{AVALIAÇÃO DA ACESSIBILIDADE UTILIZANDO O ASES}

A avaliação utilizando o software ASES resultou em 28 ocorrências (Gráfico 1) envolvendo as diretrizes de um, três e quatro.

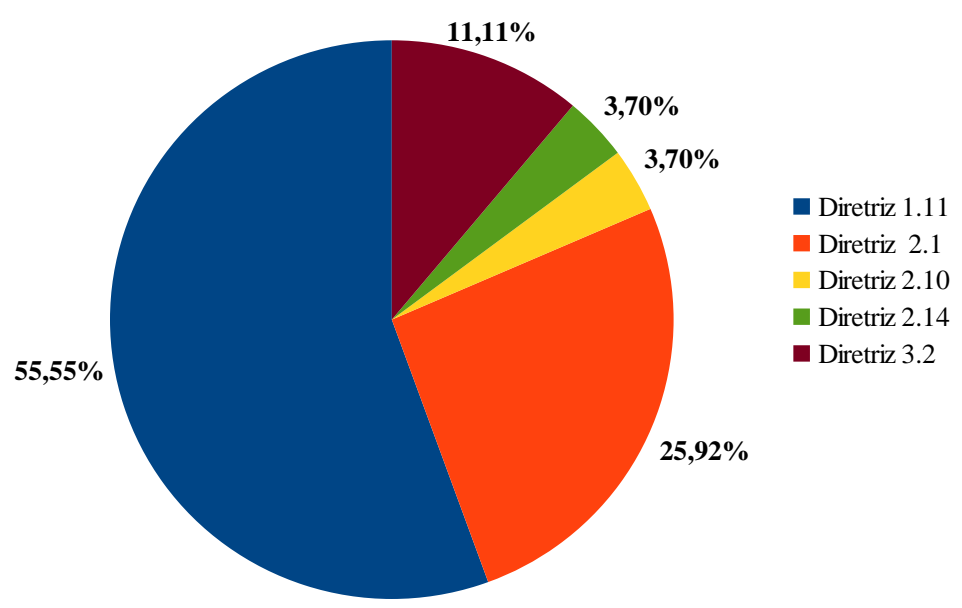

Gráfico 1 - Ocorrências encontradas utilizando o software ASES.

Pela diretriz um foram detectadas 15 ocorrências com base na recomendação 1.11 da Cartilha Técnica (de prioridade um) que adverte fornecer um equivalente textual às imagens. As ocorrências possuem o equivalente textual vazio e uma também não possuía o atributo "alt" declarado na tag "img". Abaixo, seguem três das quinze ocorrências detectadas:

- <img src="++resource++ifrn.theme.images/accessibility_icon.gif">;

- <img src="http://www.ifrn.edu.br/logo.png" alt="" title="" height="108" width="247" $1>$;

- $\quad$ <img alt=“" src="http://www.ifrn.edu.br/spinner.gif" />.

Através da diretriz três foi detectada uma ocorrência com base na recomendação 2.10

\footnotetext{
${ }^{12}$ https://www.governoeletronico.gov.br/acoes-e-projetos/e-MAG/e-mag-3-0-consulta-publica
} 
(de prioridade dois), que pede para utilizar cabeçalhos em uma ordem lógica. Abaixo segue a ocorrência detectada:

- $\quad<$ h5 class="hiddenStructure" $>$ Seções</h5>.

Ao observar o código-fonte, foi percebida uma ocorrência que o ASES não identificou em seu relatório. Ainda pela diretriz três, através da recomendação 2.10 (de prioridade dois):

- $\quad$ h5 class="hiddenStructure" $>$ Ferramentas Pessoais</h5>.

Pela diretriz três, por meio da recomendação 2.1 (de prioridade dois), foram detectadas três ocorrências. Como o elemento img é vazio, ele deve ser fechado na mesma tag em que foi aberto. Abaixo segue uma das ocorrências detectadas:

- <img src="++resource++ifrn.theme.images/sitemap_icon.gif">.

Através da diretriz quatro foi detectada uma ocorrência com base na recomendação 2.14 (de prioridade dois), pois não há caracteres pré-definidos na caixa de texto selecionada. A seguir, a linha com a ocorrência detectada:

- <input name="SearchableText" type="text" size="18" title="Buscar no Site" accesskey="4" class="inputLabel" id="searchGadget" />.

Pela diretriz quatro, por meio da recomendação 3.2 (de prioridade três), foram detectadas sete ocorrências, pelo fato de não haver sido atribuídos os elementos abbr ou acronym para as siglas encontradas no Portal IFRN. Abaixo, três destas ocorrências:

- $\quad<$ a accesskey="" href="http://www.ifrn.edu.br/rss" title="RSS"><img src="++resource++ifrn.theme.images/rss_icon.gif" $>$ RSS $</ a>$;

- $\quad<$ span class="tile">Divulgada a concorrência dos processos seletivos do IFRN</span>;

- $\quad<$ span class="local">V FNBIFs</span>.

\section{AVALIAÇÃO UTILIZANDO O SOFTWARE DOSVOX}

Através da funcionalidade de leitor de telas do software DOSVOX, foi possível detectar alguns pontos (Quadro 3) que, se corrigidos, podem tornar o Portal IFRN mais acessível às pessoas portadoras de deficiência visual. O software leu o conteúdo da página inicial do portal, então foi observado como o software retornava ao usuário o conteúdo que estava lendo. O software permitiu comprovar algumas ocorrências já encontradas na análise utilizando o ASES. 
Quadro 3: Ocorrências identificadas utilizando o software DOSVOX.

\begin{tabular}{|c|c|c|}
\hline N. & Ocorrência & Recomendações \\
\hline 1 & $\begin{array}{c}\text { O leitor de telas leu, por exemplo, } \\
\text { "accessibility_icon.gif". }\end{array}$ & $\begin{array}{c}\text { Inserir o atributo "alt" na tag do } \\
\text { elemento "img". }\end{array}$ \\
\hline 2 & $\begin{array}{c}\text { O leitor de telas leu "logo.png" em "<img } \\
\text { src="http://www.ifrn.edu.br/logo.png" } \\
\text { alt="" title="" height="108" width="247" } \\
\text { />". }\end{array}$ & $\begin{array}{l}\text { Preencher o atributo "alt" na tag } \\
\text { do elemento "img". }\end{array}$ \\
\hline 3 & $\begin{array}{l}\text { Ao ler as seções do site, os links dos campi } \\
\text { são lidos logo após as divisões do site, sem } \\
\text { nenhuma separação. }\end{array}$ & $\begin{array}{l}\text { Inserir antes do nome do primeiro } \\
\text { campus um identificador, por } \\
\text { exemplo, "Listagem dos Campi } \\
\text { IFRN", para que o usuário do leitor } \\
\text { de telas compreenda mais } \\
\text { facilmente a divisão dos links. }\end{array}$ \\
\hline 4 & $\begin{array}{c}\text { O equivalente textual atribuído a algumas } \\
\text { imagens é igual ao texto anterior ou } \\
\text { posterior a elas, fazendo com que o leitor } \\
\text { de telas repita a mesma informação duas } \\
\text { vezes. }\end{array}$ & $\begin{array}{l}\text { Remover ou modificar o texto } \\
\text { anterior ou posterior à imagem. }\end{array}$ \\
\hline 5 & $\begin{array}{c}\text { O leitor de telas lê o número romano como } \\
\text { a letra com a qual ele é representado. }\end{array}$ & $\begin{array}{c}\text { Evitar o emprego de numeração } \\
\text { romana. }\end{array}$ \\
\hline 6 & $\begin{array}{l}\text { Dificuldade de compreensão de siglas, tais } \\
\text { como IFRN e FNBIF (pois leitor de telas não } \\
\text { lê letra a letra, mas tenta ler a sigla como } \\
\text { se fosse uma só palavra) e PPP (pois não é } \\
\text { uma sigla comum e pode ser que o usuário } \\
\text { não saiba o que significa). }\end{array}$ & $\begin{array}{c}\text { Utilizar tags apropriadas para } \\
\text { descrever siglas e acrônimos como } \\
\text { "abbr" ou "acronym". }\end{array}$ \\
\hline
\end{tabular}

\section{AVALIAÇÃO A PARTIR DO WCAG SAMURAI}

Através da diretriz um do WCAG Samurai, por meio do ponto de verificação 1.1 (de prioridade um), foram detectadas 16 ocorrências (Gráfico 2). 


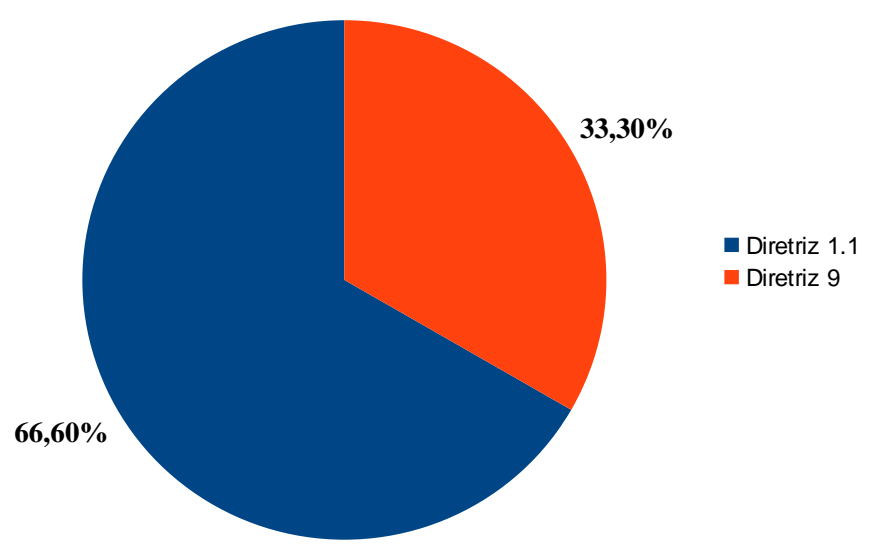

Gráfico 2 - Ocorrências encontradas com base nas diretrizes do WCAG Samurai.

Três delas em virtude de não haver equivalente textual atribuído à imagem. A seguir, uma das ocorrências:

- $\quad$ img src="++resource++ifrn.theme.images/rss_icon.gif">.

Ainda com relação à diretriz um, duas ocorrências foram detectadas por haver equivalente textual vazio sem texto anterior ou posterior cumprindo função de texto equivalente. A seguir, as duas ocorrências:

- <img src="http://www.ifrn.edu.br/logo.png" alt="" title="" height="108" width="247" $1>$;

- <img alt="" src="http://www.ifrn.edu.br/spinner.gif" />.

As demais ocorrências foram detectadas por haver equivalente textual atribuído na tag "img" transcrito exatamente como o texto anterior ou posterior à imagem, fazendo com que o mesmo texto seja lido pelo leitor de telas duas vezes consecutivamente. Abaixo, seguem três destas ocorrências:

- <img class="appIMG" src="http://www.ifrn.edu.br/reitoria/servicos/faleconosco/image_thumb" alt="Fale Conosco" />;

- $\quad$ <img class="appIMG" src="http://www.ifrn.edu.br/reitoria/servicos/webmailifrn/image_thumb" alt="Webmail IFRN" />;

- $\quad$ <img class="appIMG" src="http://www.ifrn.edu.br/reitoria/servicos/qacademico/image_thumb" alt="Q-Acadêmico" />.

Pela diretriz nove (de prioridade um) foram detectadas oito ocorrências, pois o WCAG Samurai adverte a não utilizar o atributo accesskey. A seguir, três destas ocorrências:

- $\quad<a \quad$ accesskey="2" href="http://www.ifrn.edu.br/\#documentContent">Ir para o conteúdo.</a>;

- $\quad<a$ accesskey="6" href="http://www.ifrn.edu.br/\#portlet-navigation-tree">Ir para a navegação</a>;

- $\quad<$ a accesskey="3" href="http://www.ifrn.edu.br/sitemap" title="Mapa do Site"><img src="++resource++ifrn.theme.images/sitemap_icon.gif">Mapa do Site</a>. 


\section{CONCLUSÃO}

A avaliação demonstrou que o Portal IFRN apresenta conformidade com algumas diretrizes de acessibilidade web. Ao observar os resultados obtidos a partir do modelo e-MAG, foram constatadas ocorrências que podem ser rapidamente resolvidas, tornando o portal mais acessível para todos. Isso ocorre também pelo fato do portal se basear em um software que já oferece o atendimento ao WCAG 1.0. A aderência ao e-MAG é um passo importante, uma vez que o IFRN é uma Instituição Pública Federal e que atende a um público muito grande e diverso da população brasileira. Os resultados indicam que mais da metade das ocorrências detectadas são aquelas necessárias para que seja atendido o nível de acessibilidade de prioridade um do e-MAG. As outras ocorrências estão distribuídas nos níveis de prioridade dois e três. As orientações obtidas a partir das recomendações do e-MAG foram confirmadas ao se avaliar o portal utilizando o leitor de telas.

O WCAG Samurai, como errata ao WCAG 1.0, é uma boa ferramenta para a construção de páginas web mais acessíveis. A errata é mais direta do que as diretrizes disponibilizadas pelo W3C através do WCAG, pois suas instruções determinam exatamente o que o programador deve ou não fazer. Além disso, o WCAG Samurai considera tópicos que as versões oficiais dispostas pelo W3C (WCAG 1.0 e WCAG 2.0) não percebem, o que o torna mais amplo em relação à acessibilidade, mesmo sendo apenas sugestões não oficiais. Por não ser uma orientação publicada pelo W3C, é necessário avaliar bem se a errata é a mais adequada ou não, pois vai de encontro ao WCAG 2.0. Como a nova versão do e-MAG é baseada no WCAG 2.0, este trabalho oferece uma contribuição em um processo de escolha entre o e-MAG 3.0 e o WCAG Samurai para portais que façam parte do governo.

Seguindo os princípios do e-MAG 2.0 e do WCAG Samurai, há também outras ferramentas que podem tornar o Portal IFRN mais acessível. Muitos usuários não conseguem enxergar as fontes dispostas na página por serem pequenas, então, disponibilizar ao usuário a possibilidade de aumentar ou diminuir o tamanho da fonte do conteúdo da página é uma ideia sugestiva. Mas, o usuário pode não ter sua visão adequada ao contraste "conteúdofundo de página", logo, o site poderia permitir ao usuário escolher entre diferentes tipos de contraste aquele que mais agrada à sua visão. Outra sugestão seria proporcionar ao usuário a possibilidade de modificar o layout, por exemplo, modo "expandido" e modo "centralizado".

Novas pesquisas podem ser conduzidas após o e-MAG 3.0 ser oficialmente lançado. Por exemplo, comparar o e-MAG 3.0 com os princípios do WCAG Samurai. Outro trabalho futuro seria a elaboração de padrões de acessibilidade para sites do governo brasileiro. Como os padrões são específicos e adaptados ao contexto, seria possível gerar padrões com características de diferentes modelos de acessibilidade.

\section{REFERÊNCIAS}

1. BRASIL, 2005a. eMAG, Acessibilidade de Governo Eletrônico, cartilha Técnica. Departamento de Governo Eletrônico, Brasília, DF, 14 dez. 2005. Disponível em: <>. Acesso em: 01/08/2011. 
2. BRASIL, 2005b. eMAG, Acessibilidade de Governo Eletrônico, modelo de Acessibilidade. Departamento de Governo Eletrônico, Brasília, DF, 14 dez. 2005. Disponível em: <>. Acesso em: 01/08/2011.

3. BREWER, Judy. Web Accessibility Highlights and Trends. Proceedings of the 2004 international cross-disciplinary workshop on Web accessibility (W4A) (W4A '04). ACM, New York, NY, USA, 51-55.

4. CRESWELL, J. W. Projeto de pesquisa: métodos qualitativo, quantitativo e misto. 2.ed. Porto Alegre: Artmed, 2007. 248p.

5. CLARK, JOE. Building Accessible Websites. Indianápolis: New Riders, 2002. 432p.

6. CLARK, JOE. To Hell with WCAG 2. 2006. Disponível em <http://www.alistapart.com/articles/tohellwithwcag2> Acesso em: 29/12/2010.

7. Comitê Gestor da Internet no Brasil. Dimensões e características da Web brasileira: um estudo do .gov.br. São Paulo, 2010.

8. CHISHOLM, Wendy A.; HENRY, Shawn Lawton. Interdependent Components of Web Accessibility. Proceedings of the 2005 International Cross-Disciplinary Workshop on Web Accessibility (W4A) (W4A '05). ACM, New York, NY, USA, 31-37.

9. JESCHKE, Sabina; PFEIFFER, Olivier; VIERITZ, Helmut. Using Web Accessibility Patterns for Web Application Development. Proceedings of the 2009 ACM symposium on Applied Computing (SAC '09). ACM, New York, NY, USA, 129-135.

10. KELLY, Brian; SLOAN, David; BROWN, Stephen; SEALE, Jane; PETRIE, Helen; LAUKE, Patrick; BALL, Simon. Accessibility 2.0: People, Policies and Processes. Proceedings of the 2007 international cross-disciplinary conference on Web accessibility (W4A) (W4A '07). ACM, New York, NY, USA, 138-147.

11. LARA, Silvana Maria Affonso de; WATANABE, Willian Massami; SANTOS, Eduardo Pezutti Beletato dos; FORTES, Renata P. M. Improving WCAG for Elderly Web Accessibility. Proceedings of the 28th ACM International Conference on Design of Communication (SIGDOC '10). ACM, New York, NY, USA, 175-182.

12. MARCONI, Marina de Andrade; LAKATOS, Eva Maria. Metodologia científica. 5.ed. São Paulo: Atlas, 2010. 312p.

13. MITSAMARN, Namnueng; GESTUBTIM, Waragorn; JUNNATAS, Sirilak. Web Accessibility: A government's effort to promote e-accessibility in Thailand. Proceedings of the 1st international convention on Rehabilitation engineering \& assistive technology: in conjunction with 1st Tan Tock Seng Hospital Neurorehabilitation Meeting (i-CREATe '07). ACM, New York, NY, USA, 23-27.

14. SLOAN, David; HEATH, Andy; HAMILTON, Fraser; KELLY, Brian; PETRIE, Helen; PHIPPS, Lawrie. Contextual Web Accessibility - Maximizing the benefit of Accessibility Guidelines. Proceedings of the 2006 international cross-disciplinary workshop on Web accessibility (W4A): Building the mobile web: rediscovering accessibility?(W4A '06). ACM, New York, NY, USA, 121-131. 